\title{
Nursing teamwork in the care of older people: a mixed methods study
}

Article

Accepted Version

Creative Commons: Attribution-Noncommercial-No Derivative Works 4.0

Anderson, J. E., Ross, A. J., Lim, R., Kodate, N., Thompson, K., Jensen, H. and Cooney, K. (2019) Nursing teamwork in the care of older people: a mixed methods study. Applied Ergonomics, 80. pp. 119-129. ISSN 0003-6870 doi: https://doi.org/10.1016/j.apergo.2019.05.012 Available at https://centaur.reading.ac.uk/83843/

It is advisable to refer to the publisher's version if you intend to cite from the work. See Guidance on citing.

To link to this article DOI: http://dx.doi.org/10.1016/j.apergo.2019.05.012

Publisher: Elsevier

All outputs in CentAUR are protected by Intellectual Property Rights law, including copyright law. Copyright and IPR is retained by the creators or other copyright holders. Terms and conditions for use of this material are defined in the End User Agreement.

\section{www.reading.ac.uk/centaur}

\section{CentAUR}

Central Archive at the University of Reading 
Reading's research outputs online 


\section{Nursing teamwork in the care of older people: A mixed methods study of adaptive team processes}

Anderson, J.E. ${ }^{1 *}$ Ross, A.J. ${ }^{2}$ Lim, R ${ }^{3}$. Kodate, N. ${ }^{4}$ Thompson, K. ${ }^{5}$ Jensen, H. ${ }^{6}$ \& Cooney, $\mathrm{K}^{6}$

${ }^{1}$ Florence Nightingale Faculty of Nursing, Midwifery and Palliative Care, King's College London

${ }^{2}$ Dental School, University of Glasgow

${ }^{3}$ Reading School of Pharmacy, University of Reading

${ }^{4}$ School of Social Policy, Social Work and Social Justice, University College Dublin

${ }^{5}$ School of Social Science, Liverpool Hope University

${ }^{6}$ Guy's and St Thomas' NHS Foundation Trust, London, UK

Correspondence to:

Dr. Janet Anderson

King's College London

Florence Nightingale Faculty of Nursing and Midwifery

James Clerk Maxwell Building

57 Waterloo Road

London SE1 8WA

janet.anderson@kcl.ac.uk

Keywords: Teamwork, Quality of care, Nursing, Acute Care, Care of older people 


\section{ABSTRACT}

Healthcare is increasingly complex and requires the ability to adapt to changing demands. Teamwork is essential to delivering high quality care and is central to nursing. The aims of this study were to identify the processes that underpin nursing teamwork and how these affect the care of older people, identify the relationship between perceived teamwork and perceived quality of care, and explore in depth the experience of working in nursing teams. The study was carried out in three older people's wards in a London teaching hospital. Nurses and healthcare assistants completed questionnaires $(n=65)$ on known dynamics of teamwork (using the Nursing Teamwork Survey) together with ratings of organisational quality (using an adapted AHRQ HSPS scale). A sample ( $n=22 ; 34 \%)$ was then interviewed about their perceptions of care, teamwork and how good outcomes are delivered in everyday work. Results showed that many care difficulties were routinely encountered and confirmed the importance of teamwork (e.g. shared mental models of tasks and team roles and responsibilities, supported by leadership) in adapting to challenges. Perceived quality of teamwork was positively related to perceived quality of care. Work system variability and the external environment influenced teamwork and confirmed the importance of team adaptive capacity. The CARE model shows the centrality of teamwork in adapting to variable demand and capacity to deliver care processes, and the influence of broader system factors on teamworking. 


\section{INTRODUCTION}

Despite intra and inter country differences in life expectancy, there has been a steadily rising increase in life expectancy over the last century (Leon, 2011). Alongside this population change, the demand for hospital care has been rising inexorably over recent years, especially for older people. The biggest increase in hospital admissions in the UK has been in the age group 65-84, with hospital episodes rising by more than 2 million to 6.3 million over a tenyear period to 2017 (NHS Digital, 2017). Along with increased demand there is now increased focus on the quality of care received by older people. For example, in 2001 the Standing Nursing and Midwifery Advisory Committee identified significant problems in the quality of nursing care delivered to older patients in acute hospitals (SNMAC, 2001). More recently, health and social care policy initiatives for older people in the UK have included guidelines and standards produced by the National Institute for Health and Care Excellence (NICE), Care Quality Commission, Department of Health, Royal College of Nursing and Healthcare Improvement Scotland, indicating high levels of concern for this vulnerable population. Older people in hospital are at greater risk of experiencing adverse incidents including falls, pressure ulcers, infections, adverse drug reactions, delirium and incontinence (Sari et al, 2008; Long et al, 2013). Older patients (over 65) have complex care needs and are more likely to experience clinical complications, increased length of stay, and loss of functional ability following treatment in hospital (Long et al, 2013). These challenges of caring for older people in hospital mean that it is important that we understand how nursing teamwork contributes to the quality of care they receive. Quality is defined by the Institute of Medicine (1990) as having six dimensions: safety, clinical effectiveness, patient centred, timely, efficient and equitable. In this paper we focused on safety, clinical effectiveness and patient centredness. 
Nurses deliver much of the care in hospitals and comprise $25 \%$ of the NHS workforce, with support workers, including healthcare assistants, comprising an additional 25\% (Nuffield Trust, 2017). However, nursing teams have received less research attention than specialist inter-professional teams such as cardiac arrest teams (Andersen et al, 2010), operating theatre teams (Bleakley et al, 2006) and clinical resuscitation teams (Cooper et al, 2016). There is currently evidence that good nursing teamwork is related to high job satisfaction and lower rates of missed care (Kalisch, et al, 2010), better staff retention (Lartey et al, 2014), reduced mortality (Wheelan et al, 2003) and reduced errors in care (Nadzam, 2009), but it is still not clear how teamwork is translated into practice in nursing teams, how it contributes to patient care, and what factors impede or facilitate good teamwork.

Teamwork in healthcare is the co-ordination of the work of several people towards the common goal of providing safe, high quality patient care (Salas et al., 2008a; Leonard et al., 2004). Constantly changing membership and spatially distributed team members (McComb et al., 2017) make teamwork in healthcare particularly challenging. The importance of teamwork is confirmed by studies showing that poor communication and ineffective teamworking are often implicated in adverse events (e.g., Nagpal et al, 2012). There is also emerging evidence that good teamwork is associated with improved patient care, including patient safety outcomes, staff attitudes, and positive team behaviours (Manser, 2009; Lemieux-Charles \& McGuire, 2006). However, the details of which specific aspects of teamwork influence patient care and the mechanisms by which this occurs are poorly understood (Manser, 2009), despite the existence of high-level theories of healthcare teamwork. Such theories often contain abstract descriptions of behaviour that are difficult to translate into practice (Freeman et al., 2009). 
In this study we used Salas' (Salas et al, 2005) concept of the big five key components of effective teamworking to investigate how nurses perceive teamwork and how it is enacted in practice. The five key factors are:

1. Performance monitoring: members monitor each other's performance to rectify mistakes and give feedback about performance.

2. Back-up behaviour: members back each other up by providing feedback to improve performance, assist with successful completion of a task, and provide support to complete a task if overloaded.

3. Adaptability: ability to adapt to changing conditions in the internal team environment and in the complex and changing external environment; this may require flexibility in who performs tasks, in what order and how.

4. Team orientation: members prefer working in a team and are able to improve their own performance through input from others.

5. Team leadership: ability to co-ordinate team activities, assign tasks, assess team performance, and develop team members' abilities.

One of the key factors is team leadership and the role of leaders in co-ordinating team activities is emphasised in this perspective. These factors are theorised to be supported by three co-ordinating mechanisms: a shared understanding of the team's goals; effective, closed-loop communication; and mutual trust between team members. Although this framework is well cited in the teamwork literature, there is little empirical evidence to support it. In this study we aimed to understand how well it captured the important aspects of nursing teamwork. 
We also drew on concepts from resilient healthcare theory, which argues that care is complex, healthcare environments are highly dynamic and variable, and adaptive processes are crucial to successful care delivery (Robson, 2015; Braithwaite et al, 2013). Complexity is increased by multiple competing priorities and goals, incompleteness of information on which to base decisions, and intractability of processes that cannot be specified in detail. In this view of healthcare, workers' adaptations to challenges and problems are a source of safety and system resilience (Hollnagel et al., 2015).

The importance of adaptive teams (Salas et al., 2008a; Rosen et al., 2011) is now recognised, but most research in healthcare has been conducted on teams solving discrete problems rather than managing ongoing operations, as is the case for nursing teams. Studies of adaptive teams often investigate how teams deal with the high cognitive load caused by time constraints, uncertainty and complexity (Entin \& Serfaty, 1999) and have found that adaptive teams switch from explicit to implicit co-ordination mechanisms to conserve resources and attention when tasks are very demanding (Entin \& Serfaty, 1999). Although adaptation is thought to be important, it is still not well understood. The precise nature of adaptive processes and their triggers is poorly understood as noted by Grote et al (2018) and others (for example, Christian et al, 2017). This is especially true in healthcare where there is constant pressure on nursing teams due to time and task constraints.

\section{Aims}

The aims of this study were 1) to identify the behaviours and adaptive processes that underpin nursing teamwork, and how these affect the care of older patients on hospital wards; 2) to identify the relationship between nurses' perceptions of the quality of teamwork and nurses' perceptions of the quality of care; and 3) to explore, in depth, the experience of working in nursing teams and how nurses conceptualise team processes. 


\section{METHOD}

Local Research Ethics Committee approval for the study was obtained (REF SSHL/10/1138). Participants were provided with the study information sheet, consent form and the opportunity to ask researchers questions. Participants gave informed consent to be interviewed and for the interviews to be audio taped. Return of a completed questionnaire was viewed as implicit consent for aggregation of dis-identified questionnaire data, in accordance with ethics committee recommendations. All participants were informed that they were free to withdraw from the study at any time.

\section{Setting}

The study was carried out in a London acute care teaching hospital with approximately 832 beds. There were 84 beds in the older persons' unit with three wards, and according to national quality indicators (e.g. infection rates, falls, and patient complaints) the standard of care was above average. Although a multidisciplinary team of nurses, doctors, specialist geriatricians, physiotherapists, dieticians and social workers delivers care on the unit, this study focused on the nursing team (nurses, healthcare assistants and ward clerks). There were 90 nursing staff employed on the unit.

\section{Study design}

A mixed methods design was used, in which study data were collected using questionnaires and semi-structured interviews. Questionnaires were used to assess nurses' perceptions of teamwork (Nursing Teamwork Survey) and perceptions of the quality of care (Hospital Survey on Patient Safety Culture). Semi-structured interviews were carried out to understand in more depth the nuanced nature of teamwork and the experience of working in a nursing team. Mixed methods were used to gain a deeper understanding of teamwork than would be possible using one method alone. Investigating the phenomenon from different methodological perspectives increases confidence in the findings. 


\section{Participants}

The participants were ward staff on the older people's wards in the hospital. Ninety nursing staff and healthcare assistants were eligible to participate in the study. Sixty-five staff completed questionnaires: 41 Staff Nurses, seven Senior Nurses, and 18 Healthcare Assistants (response rate $72 \%$ ).

Reported ethnicity was 40\% Black/Black British; 32\% White; 17\% Asian/Asian British; 8\% other ethnic group or mixed ethnicity and $3 \%$ preferred not to say. The age range for questionnaire respondents was 21-62 years; mean 38.8 years, SD 11.2. The average length of experience was 42 months, with a wide range from 1 month to over 14 years (SD 45.3 months).

Twenty-two of these participants were also interviewed: 13 staff nurses, two senior staff nurses, six healthcare assistants, and a ward clerk. There were 20 female and two male interviewees. Interviewees were a subset of those who completed questionnaires and volunteered to participate in interviews. Staff nurses and senior nurses differ in the amount of experience they have and their seniority. Healthcare assistants are unregistered staff who provide direct patient care under the supervision of nurses. As we wished to examine teamwork systems from multiple perspectives, non-proportional quota sampling was used to ensure that varying seniority and experience levels were represented. It has been argued that for small sample sizes (typically in studies using intensive qualitative methods), the bias from quota sampling is less detrimental to the study integrity than the lack of precision introduced by probability samples (Deville, 1991).

\section{Data collection tools}

The Nursing Teamwork Survey (Kalisch et al., 2010) was used to collect perceptions of dimensions of teamwork. This was chosen because it was based on the Big Five factors (Salas et al, 2005) and has been validated with nursing teams. It has good psychometric 
properties (Kalisch et al., 2010), assesses team members' perceptions of the functioning of the team and provides an overall view of team effectiveness as well as a means to diagnose particular problem areas. It has 33-items with five factors derived from theory (Salas et al, 2005): trust, team orientation, back-up, shared mental model, team leadership. Respondents are asked to rate each descriptive item on a 5-point scale measuring how often it occurs; $1=$ rarely, $2=25 \%$ of the time, $3=50 \%$ of the time, $4=75 \%$ of the time, and $5=$ always. The questionnaire is self-administered, and completion time was approximately 30 minutes.

Perceptions of the quality of care were assessed using the Hospital Survey on Patient Safety Culture (Agency for Healthcare Research and Quality, 2010), which we modified for this study. The original scale is composed of 12 subscales with 45 Likert scale items. For this analysis we obtained an overall measure of perceived care quality by combining two factors from the scale: Overall Perceptions of Safety (consisting of 4 items, AHRQ reported Cronbach's alpha .74); and the overall Patient Safety Grade (single item rating scale with 5 levels). To this we added a single item rating scale with five levels to assess overall perceptions of Patient Care. Cronbach's alpha for our six-item care quality measure was .72, comparable with the original 4-item measure. The measure therefore assessed quality of care including patient safety, which is a component of quality.

Semi-structured interviews were designed to explore in-depth the dimensions of teamwork assessed by the questionnaire, and other contextual factors mentioned by interviewees that affect adaptive team processes. The interview schedule was developed for this study and focused on team processes, multi-disciplinary working, organisational issues such as workload and staffing, culture and information and communication, and perceptions of patient safety and care quality. The same interview topic guide was used for all participants Survey and interview data collection proceeded in parallel. 


\section{Procedures}

The study was publicised by the researchers during briefings for ward staff. Questionnaires were subsequently distributed either by the researchers handing them directly to potential participants, or by leaving questionnaire forms and instructions on the ward. Participants could return completed questionnaires by posting them in a sealed box on the ward or handing them directly to the researchers.

Semi-structured one to one interviews were conducted in two private rooms in the hospital, and ranged in length between 20 and 60 minutes. As is common in qualitative research interview length varied according to the style and responsiveness of individual interviewees. The interview schedule was pilot tested with nurses from another area within the hospital prior to interviews taking place, with the final interview schedule incorporating amendments based on the feedback received. Nurses who participated in pilot testing did not participate in the study.

\section{Data analysis}

Questionnaires were entered into IBM SPSS v 20.0 for analysis. This included descriptive statistics, ANOVA to explore whether teamwork perceptions differed between staff groups, age, ethnicity, or the length of time participants had worked on the ward, Pearson's correlations to explore the relationship between perceptions of teamwork and perceptions of the quality of care, and multiple linear regression to examine whether perceptions of teamwork predicted perceptions of the quality of care.

Interviews were transcribed verbatim and thematically analysed using NVivo (2012) software (Braun and Clarke, 2006). Descriptive coding was first conducted by a single researcher. This involved assigning a code to segments of text reflecting the content. Data were coded deductively using the NTS factors. This was chosen because it has considerable overlap with the Big Five factors (Salas et al, 2005), but has been validated with nursing teams (Kalisch et 
al, 2010). Inductive analysis was also used to identify themes that were not contained in the NTS. Text with similar content was coded and codes were refined based on iterative cycles of coding and reviewing to ensure that the categories were coherent.

A coding framework based on the deductive and inductive analysis was then developed and applied to all the interviews. Throughout the coding process transcripts were reviewed by the whole research team to clarify the context and meaning of coded text. In testing the reliability of codes, definitions were discussed by two researchers to facilitate agreement. Both researchers were involved in study design and data collection and had a shared understanding of the codes. Minor differences were discussed between the two researchers until consensus was reached. Then an independently coded selection of meaning units (sentences or short paragraphs) into themes showed a good match with $92 \%$ agreement.

\section{RESULTS}

\section{Questionnaire results}

Cronbach's Alpha scores for the five subscales of the NTS are shown in Table 1.

Table 1 Mean Nursing Teamwork Survey subscale scores $(n=65)$

\begin{tabular}{lccc}
\hline NTS dimension & N & Mean (SD) & Reliability (alpha) \\
\hline Trust & 60 & $3.03(.88)$ & .88 \\
Team orientation & 58 & $2.96(.69)$ & .75 \\
Backup & 61 & $2.86(.78)$ & .78 \\
Shared Mental Model & 60 & $3.50(.73)$ & .85 \\
Leadership & 63 & $3.04(.92)$ & .80 \\
\hline
\end{tabular}

Nursing Teamwork Survey scores indicate how often each item occurs on a 5-point scale: $1=$ rarely, $2=25 \%$ of the time, $3=50 \%$ of the time, $4=75 \%$ of the time, and $5=$ always. 
Therefore, the obtained mean subscale scores showed that good teamwork behaviour reportedly occurred slightly more than $50 \%$ of the time (mean scores ranged from 2.96 to 3.50, see Table 1), with the score for the shared mental model factor being slightly higher than the others. Table 1 shows that the subscales had good reliability (internal consistency); respondents interpreted the individual items that make up these scales as referring to similar constructs, i.e. responded to grouped items in the same direction. Analysis of variance showed that staff group (participant type) had a significant effect on perceptions of teamwork $(\mathrm{F}=4.7$, df $65, \mathrm{p}<.05)$ and perceptions of care quality $(\mathrm{F}=5$, df $65, \mathrm{p}<.01)$. A Bonferroni post-hoc follow-up test for multiple comparisons showed that healthcare assistants had significantly more positive perceptions of both teamwork (healthcare assistants mean 3.58, nurses mean 3.02; $\mathrm{p}<.05 ; 95 \% \mathrm{CI} .1-1)$ and care quality on the unit (healthcare assistants mean 4.09 nurses mean 3.59; $\mathrm{p}<.05 ; 95 \%$ CI .08-.92) than Staff Nurses.

No effects for age, ethnicity or length of experience working on the ward were found with respect to staff perceptions. There was a strong relationship between perceptions of teamwork and perceptions of care quality: Pearson's Product Moment Correlation .699 ( $\mathrm{p}<.001)$. A multiple linear regression predicting perceived quality of care from the five sub-scale factors of the Nursing Teamwork Survey gave an adjusted R Square of .498 (standard error .48, F = $11.9 ; \mathrm{p}<.001)$, and showed that leadership was a significant predictor of perceived care quality ( $\beta .323 ; \mathrm{P}<.05$, effect size .99$)$.

\section{Interview results}

Interview data are first presented according to the Nursing Teamwork Survey categories that were used deductively to code the data. These were shared mental models, trust, back-up behaviour, and team leadership. Next, four emergent themes (adaptability, communication, task demands, and work system variability) were identified inductively and are presented 
along with their relevant sub themes. In the following sections a theme describes a commonly occurring feature of the data that may have different facets. The sub themes represent different facets of the overarching theme and show specific examples of how it occurs in practice. For each sub theme we provide further description and representative extracts from the data.

\section{Shared Mental Models}

In interviews we asked participants about their concepts of good care and good teamwork to assess to what extent they had a shared understanding about good nursing care and good teamwork. Interview data showed a high level of agreement about what constituted good care, and on team goals and responsibilities. However, respondents' mental models did not completely overlap, indicating some subjectivity in perceptions of care. Themes identified in response to questions about concepts of good care are summarised and described in Table 2.

Table 2 Mental models of concepts of good care - description of sub themes

\begin{tabular}{|c|c|c|}
\hline Sub Themes & Description & Representative response \\
\hline $\begin{array}{l}\text { Personalised } \\
\text { care }\end{array}$ & $\begin{array}{l}\text { Putting patients first and } \\
\text { treating them as } \\
\text { individuals, being aware of } \\
\text { and adapting to needs, } \\
\text { providing basic care such } \\
\text { eating and drinking, } \\
\text { making sure patients are } \\
\text { comfortable and happy, } \\
\text { paying attention to detail }\end{array}$ & $\begin{array}{l}\text { "[...] the staff is good, so we need to keep it up like that } \\
\text { because we have to put the patient first" (Interview 9, HCA) } \\
\text { "Someone who puts patients' care first [...] ensuring that } \\
\text { patients are cared for properly, that nurses are doing their } \\
\text { jobs" (Interview 4, Staff Nurse) }\end{array}$ \\
\hline $\begin{array}{l}\text { Dignity and } \\
\text { privacy }\end{array}$ & $\begin{array}{l}\text { Protecting or maintaining } \\
\text { patient dignity and } \\
\text { privacy, respecting }\end{array}$ & $\begin{array}{l}\text { "Whatever they want, you help [...] as best as you can. Make } \\
\text { sure their dignity is in focus at all times. (Interview 13, Staff } \\
\text { Nurse) }\end{array}$ \\
\hline
\end{tabular}




\begin{tabular}{|c|c|c|}
\hline & $\begin{array}{l}\text { patients, treating patients } \\
\text { like their own relatives, } \\
\text { making patients feel safe } \\
\text { and valid }\end{array}$ & $\begin{array}{l}\text { "[...] preferences they have like with hair and shaving and } \\
\text { things, obviously finding [...] privacy and dignity by closing the } \\
\text { curtains and speaking to them nicely and making sure they } \\
\text { don't get neglected [...] making sure that their needs in terms of } \\
\text { eating and drinking are being met [...]" (Interview 17, HCA) }\end{array}$ \\
\hline $\begin{array}{l}\text { Communication } \\
\text { with patients } \\
\text { and relatives }\end{array}$ & $\begin{array}{l}\text { Providing information } \\
\text { such as patients' diagnosis } \\
\text { and care plan despite } \\
\text { difficulties relating to } \\
\text { patients' variable mental } \\
\text { acuity and physical } \\
\text { condition such as hearing } \\
\text { difficulties and visual } \\
\text { problems. General social } \\
\text { chat was also seen as } \\
\text { important. }\end{array}$ & $\begin{array}{l}\text { "[...]it's mostly down again to communication, maybe we } \\
\text { haven't informed the family member or something and they've } \\
\text { caught onto that and snowballed into something else which we } \\
\text { could have averted if we had communicated with them } \\
\text { effectively enough beforehand (Interview 5, Staff Nurse) } \\
\text { "I think it can be quite difficult because they have perhaps } \\
\text { visual problems or they may have hearing aids or things like } \\
\text { that so that can be quite complex but if you take the time out to } \\
\text { speak to them clearly, to sit down with them, it is rewarding”, } \\
\text { (Interview 1, HCA). }\end{array}$ \\
\hline Holistic care & $\begin{array}{l}\text { This included the care } \\
\text { provided within the } \\
\text { hospital up to the point of } \\
\text { discharge and 'total care' } \\
\text { where the medical, } \\
\text { personal and social care } \\
\text { aspects are considered. }\end{array}$ & $\begin{array}{l}\text { "Because we care for the patient the total care, it is like [...] } \\
\text { not like caring for young people, total care completely [...] So } \\
\text { not like other patients they are more dependent on the nursing } \\
\text { care" (Interview 10, Staff Nurse) }\end{array}$ \\
\hline $\begin{array}{l}\text { Understanding } \\
\text { and empathy }\end{array}$ & $\begin{array}{l}\text { Understanding the needs } \\
\text { and wants of patients. } \\
\text { Being empathic, listening, } \\
\text { reassuring, knowing } \\
\text { patient preferences, }\end{array}$ & $\begin{array}{l}\text { "[...] just listening to see if they're upset or worried about } \\
\text { anything. And most of them will tell you. Some of them need a } \\
\text { little bit more encouragement so you need to know your patient } \\
\text { a little bit better" (Interview 12, Staff Nurse) }\end{array}$ \\
\hline
\end{tabular}




\begin{tabular}{|l|l|l|}
\hline & problems and moods. & \\
Understanding social and & \\
cultural factors. & \\
\hline
\end{tabular}

Respondents were also asked about what makes a good team. Flexible adaptation to variable conditions was key to these responses. Sub themes identified were shared goals, reciprocity, supporting colleagues, patient care, and good organisation. A good team worked together, worked towards the same goal, or helped each other in their work. In some interviews, respondents described teamwork in terms of reciprocity, where the focus for a staff member was to gain help from someone who they had previously helped:

"Well good teamwork is if you are working, for instance I am working with two or three staff then we get to know each other and we find out work, so that whenever I need to, you are available to help me” (Interview 3, HCA).

Other participants talked more specifically about working together to benefit patients, supporting each other, supporting the nurse-in-charge and sharing caseloads. Others mentioned that team members who understand their roles and can organise their own work form a good team.

“...when all my colleagues come together, and you organise yourself, if you have good organisation, you organise yourself, you know what you're doing and you get everybody involved and at the end of the day you get a good result..." (Interview 20, Staff Nurse).

\section{Trust}

Trust refers to the extent to which team members are willing to communicate ideas and concerns and able to give and receive constructive feedback. Some interviewees explicitly mentioned the importance of trusting team members and knowing their capabilities and 
reliability. Good team relationships relied on social skills to ease relationships and support each other.

"I think it is trusting each other and perhaps knowing everybody's strengths and weaknesses and so knowing that if you're newly qualified you won't be able to do certain things but if you've been here a couple of years, you'll have done that and to use those strengths." (Interview 1 Band 5 nurse).

"Sense of humour, characters, respect, honesty and the same goal ..." Interview 5 nurse "We can trust each other and we're reliable ...because sometimes I will say, it's okay I will do that after you've gone for a break and if it's not done when you come back you're disappointed". (Interview 6 nurse).

"We take our responsibilities seriously ... and we are honest with each other." (Interview 2 Nurse).

There were two main themes discussed that related to trust; speaking out and receiving feedback. The majority of respondents felt comfortable speaking out about concerns they had or reporting mistakes. They felt that senior staff members were approachable and supportive.

Nevertheless, some of those who had spoken out felt that it was a difficult experience. A minority reported not feeling comfortable speaking out, including junior nurses feeling uncomfortable about speaking with doctors, and healthcare assistants feeling not able to approach nurses. Others felt that speaking about a problem would not result in any change:

“And even sometimes if I did just say, look, I find whatever, I feel like it isn't going to be taken too well...And I've been told that there's no point saying it. So in that regard, I've been told not to bother saying it, that nothing's going to change." (Interview 12, staff nurse). 
"If something is wrong, I feel comfortable to say it, even though I won't get feedback and tell me it was okay or not. It's nice to see something wrong and raise it up and they understand, but it is nice when you do that, to get feedback" (Interview 9 HCA).

Receiving feedback about their work led some respondents to feel they were being judged, and there were reports that performance feedback had led to disagreements about roles and responsibilities.

Maybe for example if we ask a nurse to do something ... but for some reason either they don't like what you said or they don't like what you've asked them to do, they take offence to it ...but if they took a step back what I'm asking them to do is not for me it's for the patient ... (Interview 5, band 5 nurse).

A related aspect was that some staff reported that not all work was viewed as equivalent. Only certain tasks, such as giving medication, washing, dressing, and providing nutrition at mealtimes, were viewed as legitimate work. Time spent socially interacting with patients outside of these tasks was viewed negatively, as a luxury in a time-poor clinical setting. Senior staff members who spent less time providing hands-on care due to administrative demands were also sometimes not perceived to be team players.

\section{Back-up Behaviour}

Back-up behaviour refers to the extent that team members are willing to step in and assist if capacity is stretched. Themes relating to backup behaviour were time, workload, individual working and reciprocity.

Many interviewees identified problems with staff members backing each other up. General difficulties included insufficient time and a heavy workload.

"I think we all try to help each other but the pressure of time means you are always thinking what is happening with my patient while I'm helping over here....” (Interview 1, staff nurse). 
However more pervasive problems were also identified. These included a reluctance of some staff to assist other team members or to meet the needs of patients who were not assigned to them. These staff focused on their own individual work:

"You know the members of staff you can ask. I've learnt that within the first two weeks, who can I ask and who will actually help me and who won 't"' (Interview 12, Staff Nurse).

Many interviewees identified a culture of reciprocity and flexible working which involved staff only helping others who had helped them in the past, emphasising views that were elicited when respondents talked about what makes a good team. Back-up behaviour was seen as an aspect of the social relationships within the ward as well as being a vital part of providing good quality care.

\section{Team Leadership}

Team leader behaviours that are crucial to effective team functioning include structuring the team, monitoring and distributing workload and assisting when staff members are overloaded. There were two key themes identified in the interview data that related to team leadership; role clarity, and support for new members of staff. Many respondents mentioned lack of role clarity as a particular difficulty. The different roles of healthcare assistants and nurses were often unclear and the lack of clear instructions from nurses to healthcare assistants was reported to be a barrier to good teamwork:

"[...] sometimes it is unclear who is doing what. Ifeel there is a bit of confusion in terms of the nursing staff and the healthcare assistants...because healthcare assistants are supposed to be supporting the nursing staff, but that role is normally those two that clash if I may say. Yes, so sometimes the healthcare assistant thinks it isn't his job to do something, and the nurse sometimes thinks a certain role should be healthcare assistant" (Interview 4, Staff Nurse). 
Additionally, respondents reported that there was a lack of understanding of all staff roles across all grades. The lack of clear role definition was seen as adding to the workload. Interviewees in general thought that staff were well supported in their work because senior staff monitored their progress and provided support if it was necessary. They also had access to a variety of training programs:

"But I do think any of the nurses if they pick up on something, they will escalate it further or they will provide that support. If you were to say I'm struggling up this end can I have some help, they'll sort it out" (Interview 1, Staff Nurse).

\section{Adaptability}

Respondents said that being able to adapt to demand pressures and problems was a feature of good teamwork. Time efficiency was the key theme related to adaptability. The lack of time was raised by many participants at several points in the interviews as a particular challenge in providing care and adapting to problems.

"It seems so simple to say that it shouldn't need to be said but obviously in some circumstances it does need to be said ...but talking, like spending time with them, listening, obviously our time is really, really crunched into a long day but like getting information from them, speaking like when we do medication rounds, giving them personal hygiene, its just like talking to the patient, communicating with the patient." (Interview 6, Band 6 Nurse).

“Yes, time, really, because sometimes we've got so many patients, and some have to go home and you know you have to get ready. Those who are going on departure, medication, some of them down". (Interview 13, HCA).

Closer examination of the interviews showed that using time efficiently was often the problem, rather than lack of time. Unexpected events in the ward, such as a serious change in a patients' state, a patient requiring close monitoring, or staff changes, contributed to 
difficulties managing time efficiently. This suggested that the teams' ability to adapt to these events was challenged, and that re-setting care priorities following a change was not always easily achieved.

"It is a challenge sometimes, it is really a challenge to staffing at times and the workload, you find that maybe you have got a lot of patients that need more attention, like more nursing care and you find it difficult to do it properly because of staff shortages and things like that yes.... and sometimes also comes back to teamwork, you find that maybe even if there is enough staff it is quite difficult to get staff to do things [right], yes." (Interview 4, Band 5 nurse).

Confusion and disagreements about roles and responsibilities was sometimes discussed as an aspect of adaptability.

"I think it's just staff not necessarily knowing what their job role is or not taking, well and in a way taking offence to when somebody asks them to do something, a certain thing but what you're asking them to do isn't anything crazy ...but again I think it comes down to us verbalising what we want for them to be doing and what we expect them to do as their role as a nurse or as a healthcare assistant." (Interview 5, Nurse Band 6).

Adaptability was required to respond to the needs of patients that can occur at unpredictable times. It was not always possible to plan and carry out tasks in a set order.

"So it just happens this is just one of the examples anyway there are so many, it so happened there is this lady, she asked for the toilet, the bed pan [okay] and then I turned around and told the healthcare assistant, we were all of us on the same bay and told her to help the lady with her bed pan because I am doing the medication with the student nurse, and the healthcare assistant turned around and said "I will do that when I finish making the bed". I 
said "no that is less important, what is important now is assisting the patient with their bed pan .... you have to do this it is more important”. (Interview 4, band 5 nurse).

Nurses also had to consider patients' preferences for their care, which meant that work plans had to adapt.

"It could be from the patient depending on the patient depending on their mentality, like they want to carry out their own dress, now let me give an example, the patient, because of events I have seen, no, no, no I don't want to, I don't want anybody around me, I don't want the dressing to be done, so [inaudible 10.01] if the patient has said no, you can't force also." (Interview 21, Band 5 nurse).

The demands of co-ordinating with members of the wider multidisciplinary team also created the need to adapt when nurses were called away at times when other team members were available;

"The nurses they are still not there to help wash the patient because once you start to do the medication the doctors call them, the social services call that one, OT call that one and then they don't help you, and you can't wait for them because you're never finished." (Interview 9, HCA).

\section{Communication}

Communication difficulty was a theme in interviews. Interviewees gave examples of poor communication between nurses, and between nurses and healthcare assistants. They specifically mentioned miscommunication and missed information as a problem during handover, which then affected teamwork and patient care. Perceived barriers to communication were difficult to identify, with most respondents citing the fact that staff members simply do not understand what is being communicated. Others highlighted that good communication was fundamental to all aspects of the work: 
"[...] good communication is very, very important because without the communication there would be no consistency of care [...] like now we turn round to the night staff-if there is no communication they would not know what has happened during the day and what they need to do or [...] where they need to start, so good communication is very, very important for us to achieve our set goal. (Interview 21, Staff Nurse)

"Like say sometimes the doctors that were at, on nightshift the other day and they didn't hand over to the nurses a patient needed .... fluids during the night and that was very, if it's in a busy environment, you don't always have time to go and see what's written in the notes, in the medical notes ... But I started at half past six and by the time I actually looked at any of the notes was around 12 o'clock so then I, only then did I realise that a patient needed fluids because they should have verbally handed that over to the nurses. " Interview 15, Band 5 nurse The need to adapt to the needs of patients sometimes led to unpredictable task demands that prevented staff from attending team handovers and meetings where important information was communicated

"I've not attended any multi-disciplinary meetings since I've been there .... that's because I've not had the opportunity to. I did plan to go to one and then I got caught up with medication and then it had started so I didn't get, haven't had a chance to take part yet, but it's something I would like to do." (Interview 17, band 5 nurse).

Sharing information amongst multidisciplinary team members was key to providing good care.

"It's good communication with everybody if you don't know something about a patient then the physio may know more or the OT may know more so they chip in and add in or whatever..." (Interview 20, Band 5 nurse). 


\section{Task demands}

All interviewees described the challenges of caring for older patients with multiple comorbidities, sensory and cognitive deficits and complex needs. Challenges included language problems, poor hearing and vision, impaired mental acuity, physical problems relating to their medical condition such as not being able to speak, and difficult patient characteristics such as being aggressive or demanding. Sensory deficits create problems communicating with patients and this means that communication is often time consuming and it can be difficult to judge the level of understanding patients have.

"Obviously because of their age the hearing is not so good that is a big, big problem. You find even to write things down; their eyesight is not so good." (Interview 4, staff nurse)

"Mostly hearing makes communication difficult. Most patients are hard of hearing and don't come in with a hearing aid, or they do but the hearing aid is faulty. Also difficult is the patient's state of mind [...] if they are confused no matter how much you communicate, they still don't understand" (Interview 22, Staff Nurse).

Mental confusion also meant that nurses need to spend more time with patients.

"With older people it can be difficult to communicate with them, especially if they're mentally unclear or confused. And they might refuse care, which means that if they refuse to have their mouth care then they won't have their mouth care and then a relative comes and says 'Why hasn't he had his teeth brushed?', there's a lot of challenges, things like that. Some patients are demanding, or deliberately difficult, maybe attention seeking maybe rightly so, and it's important for nurses to be objective, no favourites". Interview 15, Staff nurse.

"I think we're too quick to sort of wait for an answer if they've perhaps got slower speech or difficulties communicating, we'll just, it will be too quick whereas if we sat there and listened.... if you've got patients that are very confused you just have to sit with them, if 
you've got 10 minutes spare just sit with them and you'll get a lot out of them. On nights you can as well, if they're confused, they don't sleep and I try and sit down with them ... if they're calling out you might think it's their illness but it might be they're worrying about something and you'll get that out of them". (Interview 1, Staff nurse).

Nurses were aware that if all the patient's needs were not addressed it was likely that they would need to be re-admitted following discharge.

"The difficult case I would mention is if a patient is delirious or has dementia it's quite difficult, but you need to have patience with them. High contact, just make high contact .... you communicate with them with verbally and non-verbally....actually because with care of the elderly they're prone to falls at home, osteoporosis and maybe they're diabetic... so sometimes if the care is not really good they come back to hospital." (Interview 8, staff nurse).

Individual tasks such as washing patients and chatting to them, involve many aspects of patient care and observing and assessing patients is a constant demand.

"When washing patient you have to sometimes look for pressure sores, heels, long nails, there's a lot of things to look for the patient, you can write it down and you know what the patient needs, what patient doesn't need, what can be done to make the patient look different, how can we make it for them to get more better. Even when you're washing, conversation, small talk to the patient there's a lot of things you can find out about the patient." (Interview 9, HCA).

"I think some people forget with communication with patients that, if you have five minutes and you're washing a patient for example, those five minutes you can find out all the information you need regarding them. I think that's been lost a little bit. Making the time 
you do spend talking to them more valuable. Find out, not just information, but find out how they are, any worries, concerns." (Interview 18, HCA).

Documentation and paperwork required a lot of time

"Sometimes, yeah, definitely. There is a lot of that. I think, because we do have so much paperwork and things like that to do, by the time you've finished all of that it doesn't really give you any more time [yeah] to spend" (Interview 18, Band 6 nurse).

\section{Work system variability and external factors}

Work system and external factors were identified by participants as creating particular problems for teamwork and the delivery of high-quality care, as presented in Table 3.

"It's like the nurses ... basically they run the unit, I mean ... they're here twenty-four seven and they stay permanently, whereas the doctors rotate every six months, except for the consultants. So, the ... from the nurses' perspective, it's like the nurses are kind of the allrounders on the ward, they do a bit of physiotherapy ... and they have to organise all the discharge planning and everything like that, whereas the other specialists are looking at certain aspects.” (Interview 15, Nurse).

Co-ordination with external agencies was the only theme related to the external environment. Handover to external agencies was often difficult and was compounded by a lack of understanding of the roles and responsibilities of practitioners in other parts of the health system. Challenges included the provision of discharge information to primary care practitioners and discharge planning with social services:

"[...] if they need to make an arrangement for a patient to go home we need to do so many phone calls which we have to hang on listening to the music, those things, if we can give those times to the patient then it will be better" (Interview 10, Staff Nurse). 
Table 3 also highlights that healthcare is characterised by a multiple team structure, and staff members had to co-ordinate their work with others in the nursing team and with the wider multi-disciplinary team. Patient and task characteristics shaped how work was achieved, including through multi-tasking. Staff levels, skill mix, and doctor rotations also affected how teams worked together.

Table 3 Work system variability affecting teamwork - description of sub themes

\begin{tabular}{|c|c|c|}
\hline Sub Themes & Description & Representative response \\
\hline $\begin{array}{l}\text { Multi-disciplinary } \\
\text { team planning, co- } \\
\text { ordination and } \\
\text { communication }\end{array}$ & $\begin{array}{l}\text { Work is organised across multiple } \\
\text { teams. The core team is comprised of } \\
\text { nurses and healthcare assistants. The } \\
\text { wider team comprises multi- } \\
\text { disciplinary professionals, including } \\
\text { doctors, physiotherapists and social } \\
\text { workers, with shared goals for the } \\
\text { patients. The two teams need to co- } \\
\text { ordinate and plan activities. Nurses } \\
\text { play a key role liaising and acting as } \\
\text { an information conduit. Healthcare } \\
\text { assistants are not included in MDT } \\
\text { meetings. }\end{array}$ & 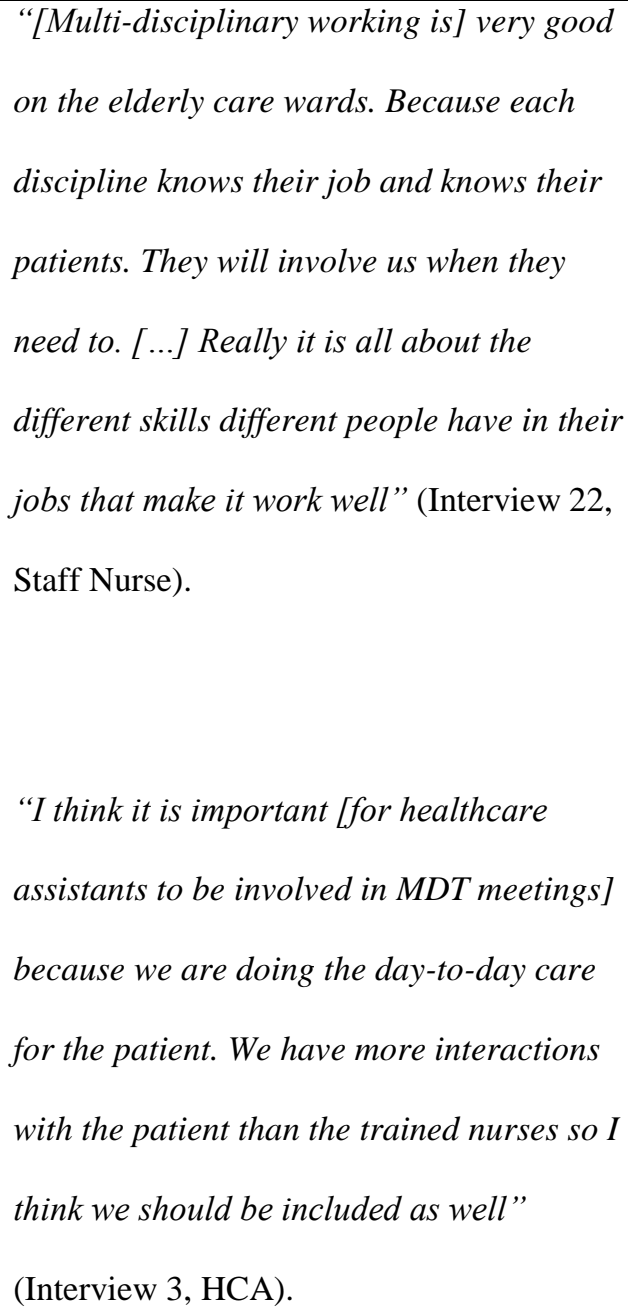 \\
\hline Staff skill levels & $\begin{array}{l}\text { Number of highly skilled staff } \\
\text { available on the ward }\end{array}$ & See above \\
\hline
\end{tabular}




\begin{tabular}{|c|c|c|}
\hline $\begin{array}{l}\text { Rotation of junior } \\
\text { doctors }\end{array}$ & $\begin{array}{l}\text { Team disruption and reduced } \\
\text { efficiency while new staff members } \\
\text { are integrated and became familiar } \\
\text { with each other's' skills and } \\
\text { knowledge }\end{array}$ & $\begin{array}{l}\text { "[...] it's like the nurses [...] basically run } \\
\text { the unit, I mean they're here twenty-four } \\
\text { seven and they stay permanently, whereas } \\
\text { the doctors rotate every six months, except } \\
\text { for the consultants" (Interview 14, Staff } \\
\text { Nurse). }\end{array}$ \\
\hline Level of staffing & $\begin{array}{l}\text { Number of staff available on the } \\
\text { ward }\end{array}$ & $\begin{array}{l}\text { "Obviously, we are short on staff, we } \\
\text { normally expect that within our ward } \\
\text { environment, but not just the amount of staff } \\
\text { but the actual expertise of the staff. There's } \\
\text { one thing to be short of staff but at least if } \\
\text { you had six staff rather than eight and they } \\
\text { were excellent [...] then you could still } \\
\text { provide really good care..." (Interview } 18 \text {, } \\
\text { Senior Staff Nurse). }\end{array}$ \\
\hline Equipment & $\begin{array}{l}\text { Unavailability of tools and patient } \\
\text { equipment seen as a patient safety } \\
\text { problem and a factor delaying } \\
\text { discharge }\end{array}$ & $\begin{array}{l}\text { "And the equipment is always breaking } \\
\text { down... Yeah, the photocopier is always } \\
\text { breaking down, then there's the stuff that } \\
\text { the patients need, that the Occupational, the } \\
\text { physios use [...] the standing hoist" } \\
\text { (Interview 7, Ward Clerk). }\end{array}$ \\
\hline Multi-tasking & Need for multi-tasking & $\begin{array}{l}\text { "Well the nurses are stretched between } \\
\text { maybe two beds and if they could have } \\
\text { maybe say four patients to one nurse maybe } \\
\text { it might be a little bit better than them } \\
\text { having one nurse to six patients and then } \\
\text { the phone keeps ringing and then they have }\end{array}$ \\
\hline
\end{tabular}




\begin{tabular}{|c|c|c|}
\hline & & $\begin{array}{l}\text { to be running from place to place" } \\
\text { (Interview } 7, \text { Ward Clerk). }\end{array}$ \\
\hline $\begin{array}{l}\text { External co- } \\
\text { ordination }\end{array}$ & $\begin{array}{l}\text { Includes handover to external } \\
\text { agencies; issues such as } \\
\text { understanding of the roles and } \\
\text { responsibilities of practitioners in } \\
\text { other parts of the health system. } \\
\text { Particular challenges included the } \\
\text { provision of discharge information to } \\
\text { GPs and discharge planning with } \\
\text { social services. }\end{array}$ & $\begin{array}{l}\text { "[...] if they need to make an arrangement } \\
\text { for a patient to go home we need to do so } \\
\text { many phone calls which we have to hang on } \\
\text { listening to the music, those things, if we } \\
\text { can give those times to the patient then it } \\
\text { will be better" (Interview 10, Staff Nurse). }\end{array}$ \\
\hline
\end{tabular}

\section{DISCUSSION}

This study, like studies of teamwork in other settings, has confirmed the importance of teamwork and leadership in delivering high quality care, as perceived and described by nursing team members. In summary, the results showed that nurses' perceptions of the quality of teamwork were aligned with their perceptions of the quality of care their patients received. That is, when nursing colleagues felt that the team was working well together, they also reported positive perceptions of the quality of care that their patients received. Perceptions of leadership were strongly related to perceptions of overall care quality and this confirms that leaders have a central role in ensuring team members work in a co-ordinated way to deliver good care. Co-ordinating complex care for older patients is challenging, and thus difficulties in team co-ordination were reported to directly affect patients and relationships between team members.

The results clearly showed the importance and challenges of nursing team adaptability in coordinating care across different shifts and different healthcare professions. These results 
augment those of previous studies which identified the importance of co-ordination between nurses and care assistants, clear communication at handover and trust in nursing teams (Kalisch et al., 2009; 2010).

The study drew deductively from Salas' five factor model of teamwork (Salas et al, 2005), and results showed high reliability for the five Nursing Teamwork Survey factors (Table 1). Although Waterson et al. (2010) have identified problems with the reliability of the AHRQ Hospital Survey on Patient Safety Culture in the UK, we found acceptable reliability for the six-item perceived care quality factor that we used (.72). There was broad agreement between the results of the Nursing Teamwork Survey and the results of the interviews probing teamwork in more depth. Nursing Teamwork Survey results indicated moderate levels of teamwork. Interview data provided a detailed picture of the dynamics and processes that lie behind the NTS scores, identified specific problem areas, and provide a promising basis for the design of training interventions to improve team functioning.

Interview and questionnaire data were broadly complementary, but there were also differences between the Nursing Teamwork Survey and interview data. The NTS contains only five of the original eight teamwork factors identified by Salas et al. (2005). Adaptability and communication, factors included in the Salas model but not part of the NTS, were identified as important in the interview data, suggesting that these should be included in future iterations of the questionnaire. There was one factor in the NTS, 'team orientation', which was not readily identified in our interview data, possibly because it is implicit in the other factors and refers to a psychological construct difficult to articulate and discuss during interviews. Nevertheless, our comparison of questionnaire and interview data strongly suggests that the NTS captures a broad and accurate overview of team functioning. 
We interpret our results as indicating the importance and the challenges of nursing team leadership and co-ordination. Nurses had high levels of agreement about the key tasks and the requirements for high quality care but reported disagreement about roles and responsibilities. Shared mental models of task requirements and team processes are required for successful team co-ordination (Fiore et al., 2010), and the lack of shared understanding of roles may have contributed to reported challenges in adapting to changing priorities and providing backup to each other. These difficulties appeared to contribute to time pressures, sporadic conflict and lack of trust between staff members. Nurses also reported the need for more explicit co-ordination from team leaders in the form of role clarification, allocation of tasks and support for new staff. This confirms previous research showing the importance of leaders in providing explicit co-ordination of work when teams are under pressure or when implicit co-ordination breaks down. Implicit co-ordination has been shown to be associated with high performance in anaesthesia teams and is associated with greater time and problem-solving efficiency (Grote et al., 2004). The team characteristics affecting implicit co-ordination are not entirely clear (Kolbe et al., 2011), but shared mental models of tasks and team roles, requiring explicit task co-ordination by the leader, appear to be important. Mediating factors such as team composition and team member experience are also likely to be important (Manser, 2009).

The theoretical framework that was used to design the study (Salas et al, 2005) emphasised the importance of team leadership in co-ordinating the team, monitoring performance and developing team members' abilities and this was supported by the findings. However, it is limited in explaining how effective team leaders put this into practice. Leadership theories from other disciplines may assist in investigating this question in more depth (for example, shared (Han et al, 2018), transformational (Eisenberg et al, 2019) or identity (Smith et al, 2018) leadership approaches may be useful). 


\section{Putting the team in situ}

Nursing teams performed a co-ordinating function within a complex structure of multiple interacting professional teams. Team members ensured there was a constant flow of information between the nursing team, doctors, and allied health practitioners, and they coordinated care for the benefit of patients. This communication and co-ordination function is crucial to patient care and requires skill and time. A multiple team structure increases the complexity of co-ordination and the opportunity for care quality problems (Kolbe et al., 2011) and so needs to be explicitly managed.

Previous studies have identified the importance of co-ordination between nurses and care assistants and have found that senior staff rate teamwork more highly than junior staff (Kalisch et al., 2009). Interestingly our HCAs rated the Unit teamwork more highly than nurses, though they also reported feeling excluded from the team and that their intimate knowledge of patients' needs was not sought or valued. Other work system factors that challenged nurses' teamwork included equipment availability, staffing levels and skill mix, doctor rotations, and task and patient factors. Co-ordination with external agencies was also problematic. These challenges required the nursing team to adapt priorities and tasks to maintain high quality care, but this was reportedly difficult and an area that could be improved. Interestingly, physical environment factors were not mentioned by interviewees. One potential explanation is that nurses are more focused on providing care and interacting with the team than on aspects of the physical environment. Further work could explore this issue as it is known that physical space is an important constraint on healthcare work (Hignett, 2001; Maben et al., 2016). 


\section{Adaptive healthcare teams}

The influence of work system factors on clinical work has been well recognised in models such as the SEIPS model (Holden et al, 2013), which emphasises the role of people in the delivery of care to a patient and identifies the role of work system factors in shaping care. Previously, we have proposed a systems model known as the CARE model (Anderson et al., 2016; see Figure 1), which captures the complexity of the environments in which nursing teams operate, and which we argue can be used as a lens to consider the present data as representing adaptive teamwork situated within a complex system.

The model shows that the work system has certain demands and capacities, which are often misaligned, as shown in our results documenting the pressures of lack of staff, inadequately skilled staff, lack of equipment, co-ordinating a multi-disciplinary team, regular staff rotations and the need to multitask. These work system pressures have also been highlighted in critiques of New Public Management, a collection of approaches to the management of public services, including cost cutting, performance management, competition and output control (for example see Diefenbach, 2009; Andrews \& Van de Walle, 2013). These misalignments must be managed by healthcare teams if they are to continue to deliver good outcomes. The ability to adapt to these conditions is essential if nurses are to deliver good care. Our data (see Table 3) showed that demands included the need to co-ordinate within a multiple-team structure and with external agencies; to multitask; and to respond to challenging patient needs, which included preferences for the way care is delivered. Capacity to respond to these needs requires sufficient staff with the necessary skill mix and available equipment, and good teamwork skills. The data showed that the ability of the team to adapt and cope with these pressures was essential for the provision of good care; breakdowns in team co-ordination and adaptation clearly resulted in difficulties, including in re-ordering priorities when the context changed. 
The CARE model, we argue, provides a means to conceptualise the demands on nursing teams and the importance of teamwork in adapting to them. Adaptive team processes have been studied extensively in other domains, such as the military (e.g. Entin \& Serfaty, 1999), and for informing team training (Salas et al, 2008b), and are better understood in other domains than in nursing teams. This study provides an overview of the importance of adaptation for nursing teams and a direction for further research to unpick the detail of how adaptive teams in nursing operate and can be improved.

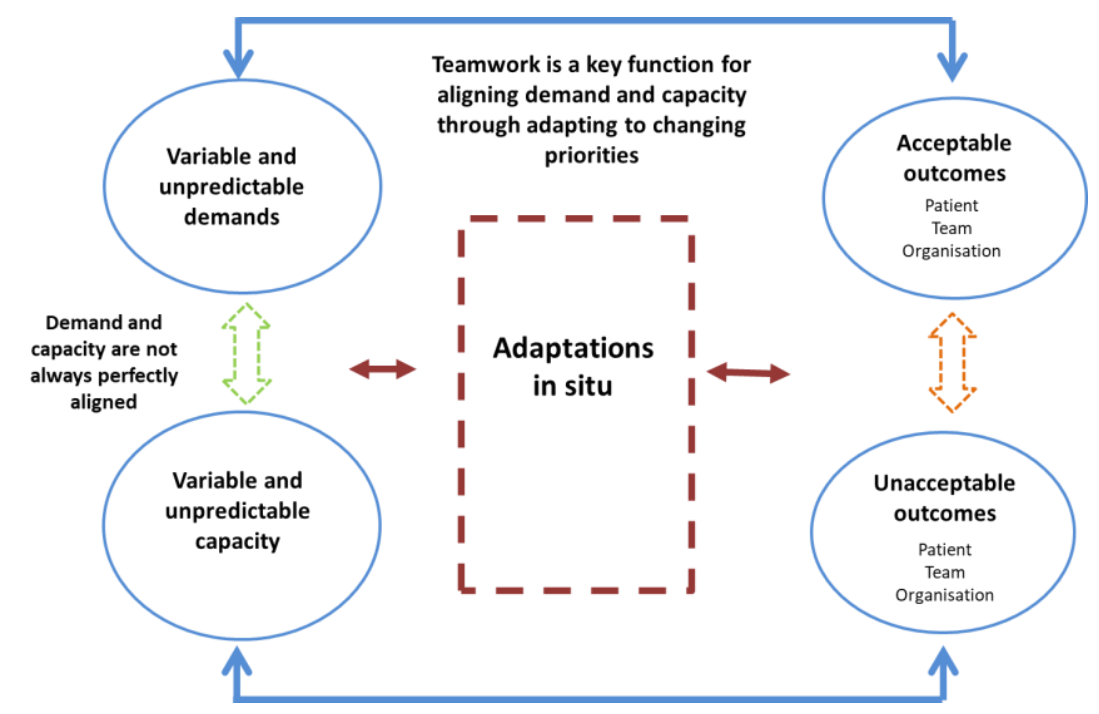

Figure 1 CARE model of adaptive performance in complex healthcare systems (Adapted from Anderson et al., 2016) 


\section{Implications of the results}

The results of the study highlight opportunities for human factors and ergonomics experts to work with nursing teams to improve non-technical skills, teamwork and leadership. Nontechnical skills are the cognitive and social skills that allow workers to communicate and coordinate their actions (Kodate, Ross, Anderson \& Flin, 2012). Although non-technical skills training using high and low fidelity simulation is common in healthcare (Rosen, 2008), the importance of adaptive capacity and the role of leaders in supporting flexible adaptation is only starting to be discussed (Paige et al, 2018) and the concept of team adaptive capacity is not common in nurse training programmes. Similarly, the role of nurse leaders in coordinating teamwork has been highlighted by this study but is not often addressed in training programmes. This study clearly shows the importance of improving teamwork, leadership and adaptive capacity in nursing teams and provides important contextual detail about the nature of nursing work and its challenges.

The results have a number of implications for quality improvement in the care of older people. First, nursing teamwork and leadership were highlighted as important influences on the perceived quality of care and efforts to improve quality should focus on strengthening these aspects of nursing work. Understanding the need for teams to adapt to difficult and changing demands, as shown in the CARE model (Anderson et al., 2016), highlights the need to strengthen this ability. Team training (Ross et al., 2012) could explicitly address adaptive teamwork, with a focus on the role of leaders in promoting team co-ordination and adaptation.

Second, the results showed that work system variability affects teamwork. Many factors created challenges for the ability of the team to meet the demands of providing care, including co-ordinating with other professionals, ensuring adequately skilled staff, available equipment and the need to multitask. Senior managers should examine how organisational 
policies and requirements can be made more supportive of effective teamwork by considering, for example, staffing levels, staff skill mix, and the workload imposed by rotation of staff and the need to co-ordinate with the wider multi-disciplinary team.

Finally, co-ordination with external agencies was difficult. This is likely to prove challenging to address because of the difficulties of cross organisational working. In recent years, policy initiatives have been taken to achieve closer collaboration between acute and communitybased care (Goodwin et al., 2014; Araujo de Carvalho et al., 2017), but future research will be required to address these co-ordination issues in practice. Acknowledgement of the difficulties involved and provision of sufficient resources to work effectively with external agencies would be desirable.

This study investigated perceptions of care and teamwork, but future human factors and ergonomics research could investigate links between teamwork and patient outcomes and include objective measures of teamwork such as in situ observations, and patient outcome data. There is also a role for longitudinal studies that explore the relationship between teamwork and outcomes over time, given the changing nature of healthcare teams. Nursing work and nursing teams have been relatively under studied in human factors and ergonomics compared to other healthcare teams, but clearly nursing work is central to patient care and worthy of further investigation. The study did not investigate the functioning of interprofessional teams, which are important in delivering high quality care for older people, and which might pose greater challenges to effective team-working than we found in the nursing teams (Ginsburg and Bain 2017; McComb et al, 2017).

\section{Conclusions}

In conclusion, this study has highlighted the importance of nursing teamwork in adapting to pressures to provide high quality inpatient care for older people. Nurses' perceptions of the quality of teamwork was positively related to their perceptions of the quality of care. 
Leadership and team co-ordination were challenging for the nursing teams, and sometimes resulted in role and task allocation confusions, and difficulty reconfiguring work goals when priorities changed. Efforts to improve teamwork should focus on developing and supporting adaptive capacity in nursing teams. Organisational factors that affect teamwork should also be addressed, including recognition of the challenges of co-ordinating multiple teams and dealing with external agencies. The CARE model (Anderson et al., 2016) provides a means to conceptualise and focus improvement efforts by showing how the demands of the work system require teams to flexibly adapt to pressures and problems to provide good care.

\section{ACKNOWLEDGEMENTS}

This work was supported by funding from Guy's and St. Thomas's NHS Foundation Trust. 


\section{REFERENCES}

Agency for Healthcare Research and Quality, 2010. Hospital Survey on Patient Safety Culture. Online http://www.ahrq.gov/qual/patientsafetyculture/ Last accessed 27th June 2012.

Andersen, P.O., Jensen, M.K., Lippert, A. and Østergaard, D. 2010. Identifying non-technical skills and barriers for improvement of teamwork in cardiac arrest teams. Resuscitation, 81(6), pp.695-702.

Anderson, J.E., Ross, A.J., Back, J., Duncan, M., Snell, P., Walsh, K. and Jaye, P. 2016. Implementing resilience engineering for healthcare quality improvement using the CARE model: a feasibility study protocol. Pilot and feasibility studies, 2(1), 61.

Andrews, R. and Van de Walle, S., 2013. New public management and citizens' perceptions of local service efficiency, responsiveness, equity and effectiveness. Public Management Review, 15(5), pp.762-783.

Araujo de Carvalho, I., Epping-Jordan, J., Pot, A.M., Kelley, E., Toro, N., Thiyagarajan, J.A. et al. 2017. Organizing integrated health-care services to meet older people's needs. Bull World Health Organ 95:756-763.

Bleakley, A., Boyden, J., Hobbs, A., Walsh, L. and Allard, J. 2006. Improving teamwork climate in operating theatres: the shift from multi-professionalism to inter-professionalism. Journal of interprofessional care, 20(5), 461-470.

Braithwaite, J. Clay-Williams, R., Nugus, P., \& Plumb, J. (2013). Health care as a complex adaptive system. In Hollnagel, E., Braithwaite, J., \& Wears, R. (Eds) Resilient Health Care. Ashgate, Aldershot. 57-76

Braun, V., Clarke, V. 2006. Using thematic analysis in psychology. Qualitative Research in Psychology 3, 77-101.

Christian, J.S., Christian, M.S., Pearsall, M.J. and Long, E.C., 2017. Team adaptation in context: An integrated conceptual model and meta-analytic review. Organizational Behavior and Human Decision Processes, 140, pp.62-89.

Cooper, S., Cant, R., Connell, C., Sims, L., Porter, J.E., Symmons, M., Nestel, D. and Liaw, S.Y. 2016. Measuring teamwork performance: Validity testing of the Team Emergency Assessment Measure (TEAM) with clinical resuscitation teams. Resuscitation, 101, pp.97-101.

Deville, J.C., 1991. A theory of quota surveys. Survey Methodology, 17(2), 163-181.

Diefenbach, T., 2009. New public management in public sector organizations: the dark sides of managerialistic 'enlightenment'. Public administration, 87(4), pp.892-909.

Eisenberg, J., Post, C. and DiTomaso, N., 2019. Team Dispersion and Performance: The Role of Team Communication and Transformational Leadership. Small Group Research, p.1046496419827376.

Entin, E.E. Serfaty, D., 1999. Adaptive team coordination. Human factors, 41(2), pp.312-325.

Fiore, S., Rosen, M., Smith-Jentsch, K., Salas, E., Legsky, M., Warner, N. 2010. Toward an understanding of macrocognition in teams: Predicting processes in complex collaborative contexts. Human Factors, 52, 203-224. 
Freeman, M., Miller, C., \& Ross, N. 2009. The impact of individual philosophies of teamwork on multi-professional practice and the implications for education. Journal of Interprofessional Care, 14:3, 237-247.

Ginsburg, L., Bain, L. 2017. The evaluation of a multifaceted intervention to promote "speaking up" and strengthen interprofessional teamwork climate perceptions. Journal of Interprofessional Care, 31(2), 207-217.

Goodwin, N., Dixon, A., Anderson, G., Wodchis, WP. 2014. Providing integrated care for older people with complex needs: lessons from seven international case studies. London: The King's Fund.

Grote, G., Zala-Mezo, E., Grommes, P. 2004. The effects of different forms of co-ordination on coping with workload. In: Dietrich, R.,Childress, T., (Eds.) Group interaction in high risk environments. Aldershot: Ashgate Publishing.

Grote, G., Kolbe, M. and Waller, M.J. 2018. The dual nature of adaptive coordination in teams: Balancing demands for flexibility and stability. Organizational Psychology Review, 8(23), pp.125-148.

Han, S.J., Lee, Y., Beyerlein, M. and Kolb, J., 2018. Shared leadership in teams: The role of coordination, goal commitment, and knowledge sharing on perceived team performance. Team Performance Management: An International Journal, 24(3/4), pp.150-168.

Hignett, S., 2001. Embedding ergonomics in hospital culture: top- down and bottom-up strategies. Applied Ergonomics 32, 61 -69.

Holden, R.J., Carayon, P., Gurses, A.P., Hoonakker, P., Hundt, A.S., Ozok, A.A. and RiveraRodriguez, A.J. 2013. SEIPS 2.0: a human factors framework for studying and improving the work of healthcare professionals and patients. Ergonomics, 56(11), 1669-1686.

Hollnagel, E., Wears, R.L. and Braithwaite, J., 2015. From Safety-I to Safety-II: A white paper. The resilient health care net: published simultaneously by the University of Southern Denmark, University of Florida, USA, and Macquarie University, Australia.

Institute of Medicine. Crossing the quality chasm: a new health system for the 21 st century. Washington DC: National Academy Press, 1990, p244.

Kalisch, B., Lee, H., Salas, E. 2010. The development and testing of the Nursing Teamwork Survey. Nursing Research 59, 42-50.

Kalisch, B., Weaver, S., Salas, E. 2009. What does nursing teamwork look like? A qualitative study. Journal of Nursing Care Quality 24, 298-307.

Kodate, N., Ross, A., Anderson, J. E., \& Flin, R. (2012). Non-Technical Skills (NTS) for enhancing patient safety: achievements and future directions. Japanese Journal of Quality and Safety in Healthcare, 7(4), 360-370.

Kolbe, M., Burtscher, M., Manser, T., Kunzle, B., Grote, G. 2011. The role of coordination in preventing harm in healthcare groups: Research examples from anaesthesia and an integrated model of coordination for action teams in health care. In: Boos, M. (Ed.) Coordination in human and primate groups, Berlin: Springer-Verlag. 
Lartey, S., Cummings, G. and Profetto- McGrath, J. 2014. Interventions that promote retention of experienced registered nurses in health care settings: a systematic review. Journal of nursing management, 22(8), 1027-1041.

Lemieux-Charles, L., \& McGuire, W. L. 2006. What do we know about health care team effectiveness? A review of the literature. Medical Care Research and Review, 63, 263300.

Leon, D. A. (2011). Trends in European life expectancy: a salutary view. International Journal of Epidemiology, 40, 271-277.

Leonard M, Graham S, Bonacum D. 2004. The human factor: the critical importance of effective teamwork and communication in providing safe care. BMJ Quality \& Safety, 13:i85-i90

Long, S. J., Brown, K. F., Ames, D., \& Vincent, C. 2013. What is known about adverse events in older medical hospital inpatients? A systematic review of the literature. International journal for quality in health care, 25(5), 542-554.

Maben, J., Griffiths, P., Penfold, C., Simon, M., Anderson, J. E., Robert, G., ... \& Barlow, J. 2016. One size fits all? Mixed methods evaluation of the impact of $100 \%$ single-room accommodation on staff and patient experience, safety and costs. BMJ Qual Saf, 25(4), 241-256.

Manser, T. 2009. Teamwork and patient safety in dynamic domains of healthcare: A review of the literature. Acta Anaesthesiology Scandinavia 53, 143-151.

McComb, S.A., Lemaster, M., Henneman, E.A., Hinchey, K.T. 2017. An evaluation of shared mental models and mutual trust on general medical units: Implications for collaboration, teamwork, and patient safety. Journal of Patient Safety, 13(4), 237-242.

Nadzam, D.M. 2009. Nurses' role in communication and patient safety. Journal of Nursing Care Quality, 24(3), pp.184-188.

Nagpal, K., Arora, S., Vats, A., Wong, H.W., Sevdalis, N., Vincent, C. and Moorthy, K. 2012. Failures in communication and information transfer across the surgical care pathway: interview study. BMJ Qual Saf, 21, 843-849..

NHS Digital 2017. Hospital admitted patient care activity 2016-17. Available online https://files.digital.nhs.uk/pdf/0/8/hosp-epis-stat-admi-summ-rep-2016-17-rep.pdf

Nuffield Trust 2017. The NHS workforce in numbers. Facts on staffing and staff shortages in England. Published online. https://www.nuffieldtrust.org.uk/resource/the-nhs-workforcein-numbers

NVivo qualitative data analysis Software; QSR International Pty Ltd. Version 10, 2012.

Paige, J.T., Fairbanks, R.J.T. and Gaba, D.M., 2018. Priorities related to improving healthcare safety through simulation. Simulation in Healthcare, 13(3S), pp.S41-S50.

Robson, R. 2015. ECW in Complex Adaptive Systems. In Wears, R., Hollnagel, E., \& Braithwaite, J. (Eds) Resilient Health Care Volume 2. The resilience of everyday clinical work. Ashgate, Aldershot.177-188. 
Rosen, M.A., Bedwell, W.L., Wildman, J.L., Fritzsche, B.A., Salas, E. and Burke, C.S. 2011. Managing adaptive performance in teams: Guiding principles and behavioral markers for measurement. Human resource management review, 21(2), 107-122.

Rosen, K.R., 2008. The history of medical simulation. Journal of critical care, 23(2), pp.157166.

Ross, A.J., Anderson, J.E., Kodate, N., Thomas, L., Thompson, K., Thomas, B., Key, S., Jensen, H., Schiff, R., Jaye, P. 2012. Simulation training for improving compassionate care of older people: An independent evaluation of an innovative programme for interprofessional education. BMJ Quality and Safety. doi:10.1136/bmjqs-2012-000954

Salas, E., Cooke, N. J., \& Rosen, M. A. 2008a. On teams, teamwork, and team performance: Discoveries and developments. Human factors, 50(3), 540-547.

Salas, E., Sims, D., Burke, C. 2005. Is there a "big five" in teamwork? Small Group Research 36, 555-599.

Salas, E., DiazGranados, D., Klein, C., Burke, C.S., Stagl, K.C., Goodwin, G.F. and Halpin, S.M., 2008b. Does team training improve team performance? A meta-analysis. Human factors, 50(6), pp.903-933.

Sari AB, Cracknell A, Sheldon TA. 2008. Incidence, preventability and consequences of adverse events in older people: results of a retrospective case-note review. Age Ageing, 37(3):265-9.

Smith, P., Haslam, S.A. and Nielsen, J.F., 2018. In Search of Identity Leadership: An ethnographic study of emergent influence in an interorganizational R\&D team. Organization Studies, 39(10), pp.1425-1447.

SNMAC. Standing Nursing and Midwifery Advisory Committee, 2001. Caring for older people: a nursing priority. DoH, London.

Waterson, P., Griffiths, P., Stride, C., Murphy, J. Hignett, S. 2010. Psychometric properties of the Hospital Survey on Patient Safety Culture: findings from the UK Quality and Safety in Healthcare 19, 1-5.

Wheelan, S.A., Burchill, C.N. and Tilin, F. 2003. The link between teamwork and patients' outcomes in intensive care units. American Journal of Critical Care, 12(6), 527-534. 